\title{
IMPLEMENTACIÓN Y PRÁCTICA DE SCRUM EN LA ASIGNATURA DE FORMULACIÓN Y EVALUACIÓN DE PROYECTOS EN LA FACULTAD DE CIENCIAS ECONÓMICAS Y ADMINISTRATIVAS DE LA UNIVERSIDAD EL BOSQUE
}

\footnotetext{
Ballesteros Quintero, Lily EL BOSQUE

PANORAMA, vol. 15, núm. 29, 2021

Politécnico Grancolombiano, Colombia

Disponible en: https://www.redalyc.org/articulo.oa?id=343967896010

DOI: https://doi.org/10.15765/pnrm.v15i29.2538
}

IMPLEMENTACIÓN Y PRÁCTICA DE SCRUM EN LA ASSIGNATURA DE FORMULACIÓN Y EVALUACIÓN DE PROYECTOS EN LA FACULTAD DE CIENCIAS ECONÓMICAS Y ADMINISTRATIVAS DE LA UNIVERSIDAD

\section{(c) (1)(20)}

Esta obra está bajo una Licencia Creative Commons Atribución-NoComercial-Compartirlgual 4.0 Internacional. 
Artículos de investigación científica y tecnológica

\section{IMPLEMENTACIÓN Y PRÁCTICA DE SCRUM EN LA ASIGNATURA DE FORMULACIÓN Y EVALUACIÓN DE PROYECTOS EN LA FACULTAD DE CIENCIAS ECONÓMICAS Y ADMINISTRATIVAS DE LA UNIVERSIDAD EL BOSQUE}

SCRUM implementation and practice in the subject "formulation and evaluation of projects" in the Faculty of Economic and Administrative Sciences of Universidad El

Bosque

PANORAMA, vol. 15, núm. 29, 2021

Politécnico Grancolombiano, Colombia

Recepción: 12 Marzo 2021

Aprobación: 15 Junio 2021

DOI: https://doi.org/10.15765/ pnrm.v15i29.2538

Redalyc: https://www.redalyc.org/ articulo.oa?id=343967896010
Implementação e prática de SCRUM na disciplina de formulação e avaliação de projetos na Faculdade de Ciências Econômicas e Administrativas da Universidad El Bosque

Lily Ballesteros Quintero laballesteros@unbosque.edu.co Universidad del Bosque, Colombia

https://orcid.org/0000-0003-3611-909X

Resumen: En los últimos años, la metodología ágil ha venido revolucionando el mundo de los proyectos (Rigby et al., 2016), y es cada vez más usada por diferentes industrias a nivel mundial (KPMG, 2019). Uno de los marcos más adoptados dentro de esta metodología es SCRUM (Kapitsaki \& Christou, 2014). Con SCRUM se busca tratar los proyectos de manera iterativa y adaptativa, brindándole flexibilidad a la toma de decisiones durante el desarrollo de los proyectos. En este estudio de caso, se realiza un análisis de la implementación y el desarrollo de proyectos con SCRUM en una facultad de ciencias económicas y administrativas, donde estudiantes de dos carreras afines (administración de empresas y negocios internacionales) aprenderán y desarrollarán sus proyectos bajo SCRUM Este ejercicio se ejecutará durante tres sprints de dos semanas, en los cuales los participantes realizan todos los eventos y entregan un producto terminado. Se encontró que los estudiantes lograron desarrollar sus proyectos de manera óptima, junto con una mejora en su forma de trabajar en equipo. Para los estudiantes, SCRUM se convierte en una nueva forma de desarrollar sus proyectos e incursionar en el mundo laboral. Finalmente, se logra una implementación satisfactoria que indica la importancia del aprendizaje de SCRUM en facultades de negocios.

Palabras clave: metodología ágil, Scrum, gestión de proyectos, eduScrum, aprendizaje ágil.

Abstract: In recent years, agile methodology has been revolutionizing the world (Rigby et al., 2016), it is increasingly used by different industries worldwide (KPMG, 2019). One of the most widely adopted frameworks within this methodology is SCRUM (Kapitsaki \& Christou, 2014). SCRUM seeks to treat projects in an iterative and adaptive manner, providing flexibility to decision-making during project development. 
In this case study, an analysis of the implementation and development of projects with SCRUM is carried out in a faculty of economic and administrative sciences, where students from two related careers (business administration and international business) will learn and develop their projects under SCRUM This exercise will run over three two-week sprints in which participants perform all ceremonies and deliver a finished product. It was found that the students managed to develop their projects in an optimal way, and a notable improvement was noticed in their way of working as a team. For students SCRUM becomes a new way to develop their projects and enter the world of work. Finally, a successful implementation was achieved indicating the importance of learning SCRUM in business schools.

Keywords: agile methodology, Scrum, project management, eduScrum, agile learning. Resumo: Nos últimos anos, a metodologia ágil tem revolucionado o mundo dos projetos (Rigby et al., 2016), sendo cada vez mais utilizada por diferentes áreas em todo o mundo (KPMG, 2019). Um dos frameworks mais adotados desta metodologia é o SCRUM (Kapitsaki \& Christou, 2014). Com SCRUM procura-se tratar projetos de forma iterativa e adaptativa, dando flexibilidade à tomada de decisões durante o desenvolvimento dos projetos. Neste estudo de caso, é realizada uma análise da implementação e desenvolvimento de projetos com SCRUM em uma faculdade de ciências econômicas e administrativas, onde alunos de dois cursos relacionados (administração de empresas e negócios internacionais) irão aprender e desenvolver os seus projetos no SCRUM Este exercício será executado em três sprints de duas semanas, nos quais os participantes realizam todos os eventos e entregam um produto acabado. Reparou-se que os alunos conseguiram desenvolver os seus projetos de forma otimizada, além de uma melhoria no trabalho em equipa. Para os estudantes, SCRUM torna-se uma nova forma de desenvolver seus projetos e entrar no mundo do trabalho. Finalmente, foi alcançada uma implementação bem-sucedida, indicando a importância da aprendizagem de SCRUM nas faculdades de negócios.

Palavras-chave: metodologia ágil, Scrum, gestão de projetos, eduScrum, aprendizagem ágil.

\section{Introducción}

Para iniciar este documento, debemos regresar a los años 90, donde se estaba revolucionando la industria del software a nivel mundial. Grandes empresas fueron creadas en ese entonces: compañías como Google, Yahoo, PayPal, etc. Los desarrolladores de software encontraban grandes problemas con las prácticas empresariales del momento. Se empezaron a revisar nuevas formas de hacer y tratar los proyectos, donde los entregables eran difíciles de entender para los equipos, sobre todo en la industria de IT. En 1993 se inicia el desarrollo de un nuevo framework para el desarrollo de procesos en cabeza de Jeff Sutherland, Jeffrey Mackenna, John Scumniotales y Ken Schwaber. Este framework, inspirado en el trabajo de Hirotaka Takeuchi y Ikujiro Nonakatomara en 1986, toma el nombre de Scrum, que hace referencia a una jugada crucial en el rugby para el éxito del equipo (Ungvarsky, 2017).

SCRUM es definido por Schwaber y Sutherland (2017), como "Un framework de trabajo por el cual las personas pueden abordar problemas complejos adaptativos, a la vez que entregar productos del máximo valor posible productiva y creativamente."(p.3). Si bien SCRUM se presenta como un framework "liviano y fácil de entender", también es "difícil de dominar”. Como lo señala Babaian (2019), a pesar de que los proyectos en SCRUM son exitosos, las empresas aún no han realizado el cambio 
a este framework, debido al cambio de cultura organizacional que esto representa.

SCRUM ha sido utilizado principalmente en la industria de IT, sin embargo, en los últimos años se han visto aplicaciones en otros sectores económicos, privados y públicos, incluyendo en este caso el sector educativo.

Schwaber y Sutherland (2017), determinan que SCRUM está basado en la teoría empirista, y que en SCRUM se utiliza un "enfoque iterativo e incremental para optimizar la predictibilidad y el control del riesgo" (p.4). Los autores señalan que la implementación de SCRUM se basa en tres pilares, nombrados a continuación:

-Transparencia: los participantes deben compartir un entendimiento de los procesos y tener una misma definición de "terminado", los procesos significativos deben ser visibles para todos los miembros del equipo.

-Inspección: se deben realizar inspecciones regulares (sin que interfieran en el trabajo), para evitar variaciones indeseadas.

-Adaptación: el equipo debe poder adaptarse en caso de que se requieran cambios luego de la inspección.

Los procesos de inspección y adaptación pueden llevarse a cabo gracias a los cuatro eventos por sprint con tiempo limitado, que se realizan dentro de SCRUM La tabla 1 resume las características principales de los eventos:

\begin{tabular}{|c|c|c|c|}
\hline EVENTO & OBJETIVO & DURACIÓN & PARTICIPANTES \\
\hline Sprint Planning & $\begin{array}{l}\text { Planear el trabajo a realizar durante el sprint. Se responde a } \\
\text { preguntas como: } \\
\text { - ¿Qué puede entregarse en el Incremento resultante del } \\
\text { Sprint que comienza? } \\
\text { - ¿Cómo se conseguirá hacer el trabajo necesario para } \\
\text { entregar el Incremento? } \\
\text { Se discute el objetivo del sprint, el backlog y el tiempo que } \\
\text { toma al equipo desarrollar cada ítem del backlog. }\end{array}$ & $\begin{array}{l}\text { Máximo 8h por } \\
\text { un sprint de } 1 \\
\text { mes }\end{array}$ & $\begin{array}{l}\text { Equipo SCRUM } \\
\text { completo: } \\
\text { Product Owner, } \\
\text { SCRUM Master, } \\
\text { Development } \\
\text { Team }\end{array}$ \\
\hline Daily SCRUM & $\begin{array}{l}\text { El Development team discute y planea el trabajo de las } \\
\text { siguientes } 24 \text { horas, respondiendo preguntas como: } \\
\text { - ¿Qué hice ayer para lograr el Objetivo del Sprint? } \\
\text { - ¿Qué haré hoy para lograr el Objetivo del Sprint? } \\
\text { - ¿Veo algún impedimento para lograr el objetivo del Sprint? } \\
\text { Igualmente se evalúa el progreso hacia el objetivo del Sprint y } \\
\text { se mejora la comunicación del equipo. }\end{array}$ & $\begin{array}{l}\text { Máximo 15m } \\
\text { diarios }\end{array}$ & $\begin{array}{l}\text { Development } \\
\text { Team } \\
\text { *El SCRUM } \\
\text { Master se } \\
\text { asegura de que el } \\
\text { Equipo pueda } \\
\text { realizar la } \\
\text { reunión. } \\
\end{array}$ \\
\hline Sprint Review & $\begin{array}{l}\text { En el Sprint review, se realiza la revisión del sprint. } \\
\text { - El Product Owner, explica los elementos del backlog } \\
\text { terminados y los pendientes con su respectivo planning. } \\
\text { - El Equipo de Desarrollo habla acerca de qué estuvo bien } \\
\text { durante el Sprint, qué problemas aparecieron y cómo fueron } \\
\text { resueltos esos problemas y realizan una demostración del } \\
\text { trabajo terminado. } \\
\text { - El grupo completo analiza los pasos a seguir, dejando } \\
\text { algunos elementos claros para el siguiente Sprint Planing }\end{array}$ & $\begin{array}{l}\text { Máximo } 4 \mathrm{~h} \text { por } \\
\text { un sprint de } 1 \\
\text { mes }\end{array}$ & $\begin{array}{l}\text { Equipo SCRUM } \\
\text { completo, } \\
\text { interesados. }\end{array}$ \\
\hline $\begin{array}{l}\text { Sprint } \\
\text { Retrospective }\end{array}$ & $\begin{array}{l}\text { Esta ceremonia busca que el equipo se inspeccione a sí } \\
\text { mismo, cómo fue el último Sprint en cuanto a personas, } \\
\text { relaciones, procesos y herramientas; } \\
\text { - Identificar y ordenar los elementos más importantes que } \\
\text { salieron bien y las posibles mejoras; y, } \\
\text { - Crear un plan para implementar las mejoras a la forma en la } \\
\text { que el Equipo SCRUM desempeña su trabajo. }\end{array}$ & $\begin{array}{l}\text { Máximo } 3 \mathrm{~h} \text { por } \\
\text { sprint de } 1 \\
\text { mes }\end{array}$ & $\begin{array}{l}\text { Equipo completo } \\
\text { SCRUM }\end{array}$ \\
\hline
\end{tabular}

\section{Tabla 1.}

\section{Eventos en SCRUM}

Elaboración propia basado en Schwaber \& Sutherland (2017).

Los equipos de SCRUM están compuestos por un SCRUM master (SM), Product owner (PO) y el Development team (DT). Esto permite que los equipos sean autoorganizados y no necesiten la dirección de personal 
externo al equipo. Este modelo permite que el equipo sea creativo, sea más efectivo y se encuentre más motivado.

Cada parte del equipo cumple con una tarea fundamental dentro del mismo. El SCRUM master debe ser el promotor de SCRUM dentro del equipo, debe apoyar y dar a conocer los preceptos de SCRUM dentro del equipo y la organización. Es el veedor de la realización de los eventos y ayuda a que el equipo no tenga problemas durante los sprint. El Product owner es el responsable de la maximización del valor del producto resultante, es el único responsable del manejo del backlog. El Development team es el equipo que va a desarrollar el incremento del producto terminado, debe tener la capacidad de autoorganizarse y de ser multifuncional. El equipo de desarrollo debe estar conformado entre tres y nueve personas, para que el trabajo sea productivo y ágil a la vez (Schwaber \& Sutherland, 2017).

Como lo indica Babaian (2019), "debido a que se ha utilizado con éxito para integrar una mentalidad ágil en el desarrollo y la entrega de productos complejos en diversas industrias, SCRUM es un marco convincente para cualquier organización.” (p.11).

Según Vogelzang et al. (2020), SCRUM es cada vez más usado en ambientes académicos. Es aplicado tanto a nivel de educación superior, como en los últimos grados de la educación elemental. La aplicación de metodologías ágiles puede verse en ejemplos como eduScrum, Blueprint y aprendizaje ágil (Kuz et al., 2018).

Por ejemplo, a nivel de formación académica, en ingeniería de sistemas, Cico et al. (2020) encontraron que la tendencia más popular en educación es el desarrollo de software ágil, seguida de Implementación de software, Usabilidad y Valor. Dentro de las metodologías agiles, encontraron que la práctica de SCRUM es de la más comunes. Por otro lado, Hidalgo (2019) exploró la adopción de métodos ágiles para la gestión de proyectos en iniciativas de investigación colaborativa, obteniendo como resultado que la adopción de estas metodologías, en especial el framework SCRUM "requiere un alto grado de flexibilidad y un enfoque de aprender haciendo". Finalmente, Vogelzang et al. (2020) revisan la perspectiva de la utilización de SCRUM en una clase de química en la secundaria.

\section{Método}

\section{Configuración y participantes}

Este artículo se realiza bajo la metodología de investigación de estudio de caso. Se presentó una propuesta de introducción de las metodologías agiles en la Facultad de Ciencias Económicas y Administrativas de la Universidad El Bosque. Se revisaron varias propuestas para el ejercicio, determinando que la más factible y realizable consistía en la creación de equipos de proyectos en la asignatura de Formulación y evaluación de proyectos. Esta asignatura está contenida dentro de la malla curricular de dos de los programas de la Facultad. Administración de empresas y Negocios internacionales. 
Dentro de esta asignatura se realiza la revisión de las teorías tradicionales de manejo de proyectos, notablemente el desarrollo de proyectos en cascada. En este caso, se pretendía realizar un curso básico en Moodle de manera virtual para que un grupo de estudiantes pueda capacitarse en el framework SCRUM y las metodologías ágiles, dentro de la asignatura.

Para informar a los estudiantes de esta nueva implementación, en primer lugar, se explicó por qué y cómo se implementaría SCRUM en la asignatura. En segundo lugar, cómo deben realizar su trabajo de preparación (modalidad virtual) (Vogelzang et al., 2020) y cómo debe ser el desarrollo de sus proyectos. En total se contó con la participación de 64 estudiantes de ambos programas.

Estas temáticas serían revisadas por los estudiantes durante las primeras dos semanas. Luego de estas dos semanas, los estudiantes tendrían una semana más para escoger sus equipos (cinco a nueve estudiantes), determinar el rol de cada uno dentro del equipo de SCRUM (SM, PO y DT), y finalmente iniciar sus proyectos en la semana 4. Se determinó que los proyectos debían ser desarrollados en tres sprints de dos semanas. A continuación, se presenta la planeación completa de las actividades:

\begin{tabular}{|c|c|c|}
\hline FECHA & TEMÁTICA & ACTIVIDAD ESTUDIANTES \\
\hline S1 & $\begin{array}{l}\text { Metodologías ágiles, SCRUM (usos, valores, } \\
\text { pilares). El equipo en SCRUM (roles, SM, PO, } \\
\text { DT). }\end{array}$ & $\begin{array}{l}\text { Revisar y analizar los contenidos del aula virtual. Enviar las } \\
\text { preguntas o dudas. }\end{array}$ \\
\hline S2 & $\begin{array}{l}\text { Eventos en SCRUM (sprint, sprint planning, } \\
\text { daily scrum, sprint review, sprint } \\
\text { retrospective), artefactos (backlog, product } \\
\text { backlog, incremento del producto), } \\
\text { definición de terminado. }\end{array}$ & $\begin{array}{l}\text { Revisar y analizar los contenidos del aula virtual. Enviar las } \\
\text { preguntas o dudas. }\end{array}$ \\
\hline S3 & Organización de equipos e inicio de sprint. & $\begin{array}{l}\text { Basados en el documento de instrucciones, se debe } \\
\text { realizar la organización de los grupos. Los grupos deben } \\
\text { estar conformados por mínimo cinco, máximo nueve } \\
\text { estudiantes. Se debe escoger alguno de los proyectos de la } \\
\text { lista y crear el backlog. Elegir los roles para cada uno de } \\
\text { los miembros del equipo. Finalmente, registrar la } \\
\text { información en el documento de registro. }\end{array}$ \\
\hline S4 -S5 & $\begin{array}{l}\text { Primer sprint: } \\
\text { Elegir el product backlog del sprint. } \\
\text { Desarrollar los eventos (sprint planning, daily } \\
\text { scrum, sprint review, sprint retrospective). }\end{array}$ & $\begin{array}{l}\text { Depositar en el aula virtual las evidencias, avances } \\
\text { realizados y los incrementos logrados durante el sprint. }\end{array}$ \\
\hline S6-S7 & $\begin{array}{l}\text { Primer sprint: } \\
\text { Elegir el product backlog del sprint. } \\
\text { Desarrollar los eventos (sprint planning, daily } \\
\text { scrum, sprint review, sprint retrospective). }\end{array}$ & $\begin{array}{l}\text { Depositar en el aula virtual las evidencias, avances } \\
\text { realizados y los incrementos logrados durante el sprint. }\end{array}$ \\
\hline S8-S9 & $\begin{array}{l}\text { Primer sprint: } \\
\text { Elegir el product backlog del sprint. } \\
\text { Desarrollar los eventos (sprint planning, daily } \\
\text { scrum, sprint review, sprint retrospective). }\end{array}$ & $\begin{array}{l}\text { Depositar en el aula virtual las evidencias, avances } \\
\text { realizados y el incremento final, el desarrollo total del } \\
\text { proyecto elegido. }\end{array}$ \\
\hline
\end{tabular}

Tabla 2.

Planeación de actividades.

Fuente: elaboración propia (2021).

En la semana 3, los estudiantes deben escoger sus grupos de acuerdo con la norma de Scrum, en donde el equipo debe estar conformado por mínimo tres y máximo nueve personas, ya que debe ser lo suficientemente pequeño para seguir siendo ágil y lo suficientemente grande como para completar un trabajo significativo dentro de un Sprint (Schwaber \& Sutherland, 2017). Sin embargo, siendo un ejercicio académico de grupos grandes se determinó que el grupo debía ser mínimo cinco y máximo nueve estudiantes. Para la escogencia de los proyectos, cada equipo debía 
escoger su proyecto dentro de una lista determinada, los proyectos no eran excluyentes, es decir, varios grupos podían escoger el mismo proyecto. Finalmente, debían escoger sus roles dentro del equipo, como lo indican Schwaber \& Sutherland (2017), los equipos deben ser auto organizados y multifuncionales, respetándose entre sí, para ser capaces e independientes. Esta semana también deberían crear el backlog del proyecto.

De las semanas cuatro a la nueve, se realizarían los proyectos incluyendo todos los eventos que indica SCRUM Para la realización del daily $S C R U M$ se indicó a los estudiantes que podrían realizarlo con una menor frecuencia, debido a los otros compromisos con las demás asignaturas del plan de estudios de programa.

Por otro lado, al finalizar los proyectos se aplicó a los estudiantes una encuesta, con preguntas abiertas y cerradas, con el fin de conocer su percepción de la importancia de Scrum, sus limitaciones y sus preferencias.

\section{Resultados}

Se notó una predilección en el tema de construir una e-shop; al indagar, se encontró que los estudiantes estaban más familiarizados con proyectos de este tipo gracias a la asignatura de e-commerce, en la que aprendieron cuáles eran los procesos básicos para desarrollar este tipo de negocios.

Algunos de los estudiantes solicitaron tutorías para aclarar diversos temas sobre la metodología. Si bien se habría indicado desde el principio que todas las dudas se podrían enviar por email y que serían respondidas con brevedad, se optó por realizar una sesión para aclarar dudas en tiempo real. Las principales dudas durante la sesión fueron sobre el manejo del backlog durante los sprints, y sobre el inicio y la finalización de cada sprint.

\section{Sprint 1}

El primer sprint estuvo marcado por la falta de experiencia de los estudiantes. Si bien se explicó qué debían contener las entregas, la mayoría de trabajos estuvieron incompletos o con errores de ejecución en los eventos. Durante el sprint se resolvieron dudas a cinco de los grupos por correo electrónico. Uno de los problemas más comunes encontrados fue el desarrollo de los dailys, pues los estudiantes no logran ejecutar este evento de manera adecuada, ni dentro del tiempo acordado. Al finalizar cada envío, se realizaba una retroalimentación sobre qué podría mejorarse en el siguiente sprint. Vale la pena resaltar que dos de los grupos no realizaron el envío del primer sprint.

\section{Sprint 2}

Durante el segundo sprint, los estudiantes no tuvieron dudas a lo largo del desarrollo de sus proyectos. La mayoría de grupos comprendieron la dinámica y enviaron sus entregas de manera apropiada, incluyendo las 
recomendaciones enviadas en la retroalimentación. Sin embargo, todavía se encontraban dudas sobre la ejecución de los dailys. En la mayoría de los casos, utilizaban este evento para planear algunas actividades, pero no para realizar las inspecciones del DT y del desarrollo del proyecto. Este evento vuelve a explicarse en la retroalimentación, junto con algunas recomendaciones de organización de los documentos. En esta oportunidad, un grupo no realizo el envío.

\section{Sprint 3}

Siendo el último sprint, los estudiantes trabajaron de forma adecuada y constante, los envíos mejoraron considerablemente. Siendo el último sprint, el desarrollo del proyecto debía estar completado al 100\%, y la totalidad del backlog terminado. Los estudiantes presentaron sus proyectos terminados y cumplidos. Si bien, la realización de los dailys mejoró, continuaba teniendo algunos problemas. Dos grupos no realizaron el envío programado. Durante la retroalimentación, los estudiantes demostraron estar motivados y satisfechos por aprender una nueva forma de desarrollar los proyectos. "la verdad nos emociona saber que hemos hecho las cosas bien", "con mi equipo hablamos y aprendimos mucho con esta nueva metodología”, "muchísimas gracias, aprendimos mucho con este proyecto".

Uno de los eventos que más llamó la atención a los estudiantes fue el sprint retrospective. Este evento permitió a los estudiantes inspeccionarse a ellos mismos como equipo. Dentro de sus respectivas retrospectives, los estudiantes enunciaron: "los integrantes del DT (developmentteam) descubrieron que había miembros que no tenían la creatividad y actitud necesarias para cumplir con sus tareas. La conclusión de este descubrimiento fue que el DT cumplió su objetivo de conocerse y entender sus aptitudes, que es parte de pertenecer a un equipo. Sin embargo, muchas cosas tienen que mejorar para tener un avance significativo en el segundo sprint. "Poseemos un grupo de trabajo bastante disperso, pero colaborador en su totalidad, pudiendo incrementar el alcance de los resultados a través de dinámicas recreacionales al interior del equipo"; "en mi opinión, me he sentido bastante alejado de mis compañeros, consideraría que fuera mucho mejor tener más acercamiento hacia ellos; en cuanto al desempeño, solo puedo decir que estoy a gusto, sobre todo, cómodo"; "me gusta nuestro desempeño, considero que el compromiso deberá primar ante cualquier tipo de diferencia laboral, e inclusive, trascendiendo al ámbito personal"; "el interés del equipo como siempre se notó en cada una de las actividades logrando cumplir así cada una de las expectativas... finalmente se culminó el proyecto de la mejor manera alcanzando los resultados esperados".

Al finalizar los proyectos, los estudiantes respondieron a una encuesta sobre su percepción al aprendizaje de esta nueva forma de trabajar proyectos. En total se obtuvieron 48 respuestas, ya que los grupos que no realizaron la finalización y entrega de los proyectos no participaron de la encuesta. 
Al preguntar a los estudiantes sobre la dualidad de la utilización de Scrum, tanto a nivel profesional como a nivel personal, el 91,7\% (44) respondió que consideran que SCRUM puede ser útil en ambos escenarios, mientras que el 8,3\% (4) respondió que no. Sin embargo, cuando se les pregunta si consideran que obtener una certificación en SCRUM les permitiría mayores oportunidades de trabajo, el 93,8\% (45) responde que sí, mientras que el 6,3\% (3) que no. En este punto vemos una diferencia en el uso de SCRUM en escenarios personales y profesionales, ya que de los cuatro estudiantes que habían respondido negativo en la primera pregunta, tres indican que la certificación podría ser positiva para sus oportunidades laborales, mientras que un estudiante no encuentra la utilidad de SCRUM en ningún escenario. Por otro lado, dos de los estudiantes que respondieron positivamente a la primera pregunta indican que la certificación no tendría impacto en sus oportunidades laborales.

En la parte teórica y práctica de Scrum, se les preguntó a los estudiantes cuáles de los conceptos les quedaron totalmente claros al finalizar los proyectos, conceptos como el sprint, la planeación y los roles fueron los más claros para los estudiantes, mientras que los eventos, como la retrospective y la review tuvieron menos claridad.

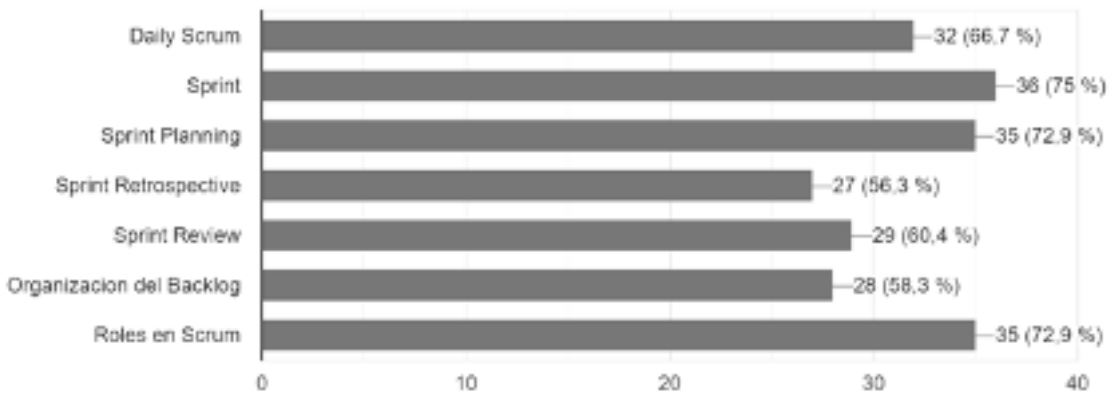

Figura 1.

Entendimiento de los conceptos por parte de los estudiantes. Fuente: elaboración propia (2021)

Se preguntó de forma abierta cuál había sido la parte más difícil durante el ejercicio. Siete de los estudiantes no encontraron ningún tipo de dificultad en la realización de sus proyectos; por otro lado, diez de los estudiantes encontraron dificultad de trabajar en equipo, seguido de seis estudiantes con problemas en el manejo del tiempo y seis en el manejo de la virtualidad. Solo un estudiante indica tener problemas con entender la teoría. 


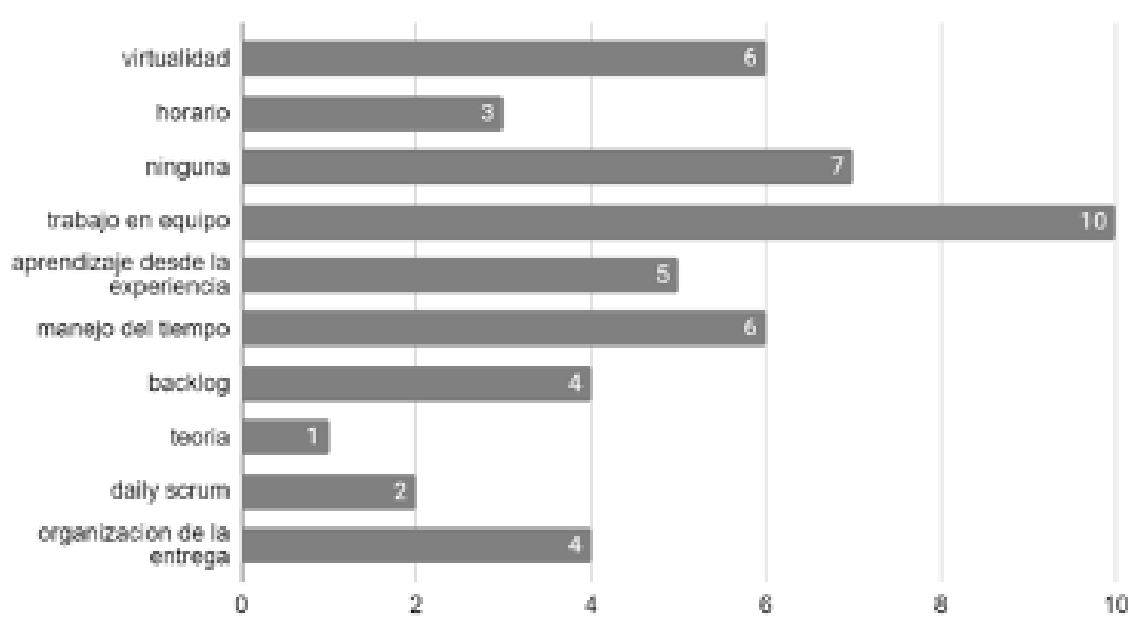

Figura 2.

Dificultades encontradas por los estudiantes.

Fuente: elaboración propia (2021)

Al preguntar si SCRUM permite una facilidad de trabajo en equipo, el 83,3\% (40) responde afirmativamente, mientras que el 16,7\% (8) responde que no. Sin embargo, solo dos de los diez estudiantes que respondieron en la pregunta anterior que su dificultad era el trabajo en equipo indican que SCRUM no les facilita el trabajo en equipo, mientras que los ocho restantes indican que sí lo facilita.

Finalmente, se pregunta a los estudiantes cuál es su preferencia de ambiente de aprendizaje para este tipo de temática; el 70,8\% (34) de los estudiantes, señala tener una preferencia de ambiente de aprendizaje mixto, en donde el trabajo práctico se realice virtualmente, pero que se dejen algunos espacios presenciales para el aprendizaje de la metodología.
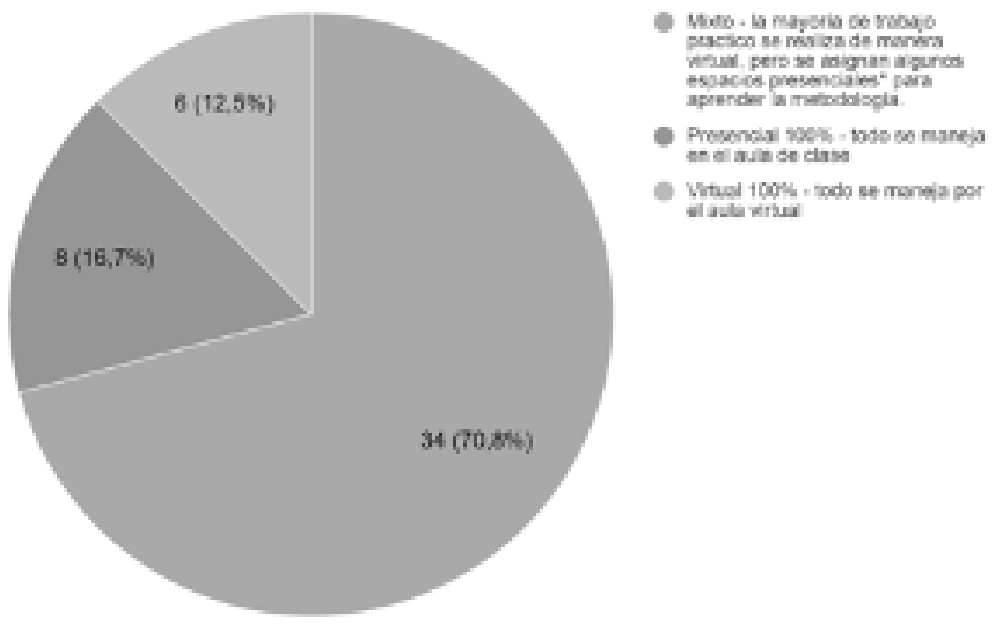

Figura 3.

Preferencia de ambiente de aprendizaje

Preferencia de ambiente de aprendizaje. Fuente: elaboración propia (2021) 


\section{Discusión y conclusión}

El desarrollo e implementación de nuevas metodologías, teorías, tecnologías, etc., debe ser fundamental en el aula de clase, como lo indican Kuz et al. (2018). Los estudiantes necesitan desarrollar capacidades, habilidades y aptitudes que les serán de utilidad en el entorno académico y luego en su carrera profesional. En nuestro caso, la implementación y práctica de SCRUM dentro de la asignatura de Gerencia de proyectos, nos permite entender cómo es el desarrollo y la percepción de este nuevo framework entre los estudiantes. Como lo señala Kuz et al. (2018), SCRUM en educación permite llevar al alumno a un papel donde asume su propio aprendizaje y puede determinar el tiempo que prepara y dedica para estudiar.

En cada uno de los sprint se vio un avance en el aprendizaje práctico de la metodología. Los proyectos fueron tomando forma y los equipos mejorando en términos de organización y comunicación. El aprendizaje se convierte en una acción de colaboración, en la que la asimilación del individuo afecta y contribuye al éxito del aprendizaje permitiendo el crecimiento de todo el equipo (Kuz et al., 2018). Esto se vio representado sobre todo en la sprint retrospective, donde los estudiantes se inspeccionaron a sí mismos y como grupo para analizar cómo había sido su desempeño y qué cambios deberían realizarse para mejorar. En este ejercicio, como en la investigación de Kuz et al. (2018), se encontró que la revisión en cada sprint permite monitorear en tiempo real el avance de los estudiantes, cómo trabaja cada equipo y solucionar problemas específicos.

A nivel de implementación de Scrum, se optó por transmitir las enseñanzas, sobre todo por medio de la práctica, ya que la integración de métodos y prácticas ágiles requiere un alto grado de flexibilidad y enfoques de "aprender haciendo" (Lauren, 2018, p.30, como se citó en Hidalgo, 2019). Esto permitió a los estudiantes interiorizar los procesos para obtener un mejoramiento continuo en cada sprint.

Es importante recalcar que el $83 \%$ de los estudiantes no tenían conocimiento, no habían escuchado sobre las metodologías ágiles, ni de Scrum, metodologías que vienen siendo más y más usadas por empresas globales que experimentaron con modelos de cascada, pero que actualmente, están tratando de mejorar sus procesos existentes para acelerar la participación del equipo con Scrum (Cristal et al., 2008). Actualmente, SCRUM está presente en todo tipo de sectores, como el desarrollo de software, industrial, de entretenimiento y bancario, como es el caso de 3M, IBM, ANZ Banking Group, Google, Spotify, (Ajmal, 2018), entre muchos otros. El conocimiento de SCRUM permitirá una mayor empleabilidad de los estudiantes al momento de egresar de sus programas, ya que su conocimiento de manejo de proyectos no se limita a las estructuras en cascada, sino también al manejo de proyectos en metodologías ágiles. Es claro también para los estudiantes que el aprendizaje y posterior certificación en SCRUM puede serles de gran utilidad en el momento de buscar trabajo. 
Por otro lado, los estudiantes tienen una preferencia a manejar cierta autonomía y poder desempeñar sus proyectos de manera independiente. Sin embargo, todavía necesitan ciertos parámetros a nivel de organización y presentación de resultados; buscan formatos, normas, patrones a utilizar para que sus resultados puedan ser comparables entre ellos. Esto puede llevarlos a tener la necesidad de un ambiente de aprendizaje mixto, en donde puedan tener su autonomía, pero con algunas sesiones donde el docente pueda atender sus dudas, inquietudes, comentarios en tiempo real.

Este ejercicio nos permitió implementar SCRUM satisfactoriamente en dos programas de la facultad, generando en los estudiantes nuevas formas de acercamiento a los proyectos. Al igual que en la investigación de Hidalgo (2019), se facilitó la generación de nuevas dinámicas de colaboración, nuevas formas de trabajar en equipo y de compartir las tareas. Por otro lado, demuestra la importancia en la enseñanza de metodologías ágiles en las facultades de negocios, donde su formación como futuros gerentes, consultores, etc., les permitirá desempeñar diferentes roles con la visión holística de este tipo de formación. Si bien la implementación de SCRUM aumenta inicialmente la complejidad del entorno de aprendizaje (Vogelzang et al., 2020; Trujillo-Flórez, 2019, 2020), permitirá, como en el caso expuesto, obtener resultados satisfactorios, tanto para el cuerpo docente como para los estudiantes.

\section{Referencias bibliográficas}

Ajmal, S. (2018). How Agile SCRUM Training Transformed These 5 Companies. Quickstar.Com. https://www.quickstart.com/blog/how-agile-scrum-trai ning-transformed-these-5-companies/

Babaian, A. (2019). Becoming Agile with the SCRUM Framework. Software Quality Professional, 22(1), 23. edb.

Cico, O., Jaccheri, L., Nguyen-Duc, A., \& Zhang, H. (2020). Exploring the intersection between software industry and Software Engineering education-A systematic mapping of Software Engineering Trends. Journal of Systems and Software, 110736. https://doi.org/10.1016/j.jss.20 20.110736

Cristal, M., Wildt, D., \& Prikladnicki, R. (2008). Usage of SCRUM Practices within a Global Company (p. 226). https://doi.org/10.1109/ICGSE.200 8.34

Hidalgo, E. (2019). Adapting the SCRUM framework for agile project management in science: Case study of a distributed research initiative. Heliyon, 5(3), e01447. https://doi.org/10.1016/j.heliyon.2019.e01447

Kuz, A., Falco, M., \& Giandini, R. (2018). Comprendiendo la Aplicabilidad de SCRUM en el Aula: Herramientas y Ejemplos. Revista Iberoamericana de Tecnología en Educación y Educación en Tecnologia, 21, 62-70. https://d oi.org/10.24215/18509959.21.e07

Schwaber, K., \& Sutherland, J. (2017). The SCRUM Guide. The Definitive Guide to Scrum: The Rules of the Game. SCRUM org. https://www.scrumguides .org/docs/scrumguide/v2017/2017-Scrum-Guide-US.pdf\#zoom=100 
Trujillo-Flórez, L. M. (2020). Experiencias de innovación educativa - Tomo 4. Catálogo Editorial, 1(710), 1-257. https://doi.org/10.15765/poli.v1i71 0.2024

Trujillo-Flórez, L. M. (2019). Experiencias De Innovación Educativa - Tomo 2. Catálogo Editorial, 1(000), 1-128. https://doi.org/10.15765/poli.v1i00 0.2047

Ungvarsky, J. (2017). SCRUM En Salem Press Encyclopedia. Salem Press; ers. https://ezproxy.unbosque.edu.co/login?url=https://search.ebscohos t.com/login.aspx?direct=true\&db=ers\&AN=125600308\&lang=es\&sco pe $=$ site

Vogelzang, J., Admiraal, W., \& Van Driel, J. (2020). A teacher perspective on SCRUM methodology in secondary chemistry education. Chemistry Education Research and Practice, 21(1), 237-249. https://doi.org/10.103 9/C9RP00111E 